EUROPEAN ORGANIZATION FOR NUCLEAR RESEARCH

CERN-SL-2001-061 AP

CERN-NUFACT Note 99

\title{
Summary of WG3: Accelerator Studies and R\&D
}

\author{
E. Keil
}

\begin{abstract}
This report contains a summary of the activities of Working Group 3: Accelerator Studies and R\&D at NuFact'01, the 3rd International Workshop on Neutrino Factories Based on Muon Storage Rings, held in Tsukuba, Japan, from 24 to 30 May 2001.
\end{abstract}

Invited paper at NuFact'01, Tsukuba, Japan, 24 to 30 May 2001

Geneva, Switzerland

November 2, 2001 


\section{INTRODUCTION}

WG3: Accelerator studies and R\&D had three chairmen: S. Kamada KEK, E. Keil CERN and M. Zisman LBNL. Four sessions were held, on (i) Acceleration and FFAG Machines, (ii) Targetting and Collection, (iii) Cooling, and (iv) Proton Drivers and Other Topics, with 9, 7, 11, and 7 talks, respectively.

In the main body of this summary, I shall highlight new developments session by session, but I shall not attempt a complete survey.

Table 1: Neutrino factory parameters

\begin{tabular}{|c|c|c|c|}
\hline & Europe & Japan & US \\
\hline Proton source & $\mathrm{H}^{-}$Linac & Synchr. & Synchr. \\
\hline Energy/GeV & 2.2 & 50 & 24 \\
\hline Beam power/MW & 4 & 1 & 1 \\
\hline Repetition rate/s ${ }^{-1}$ & 50 & 0.5 & $2.5 \cdot 6$ \\
\hline Proton flux $/ \mathrm{s}^{-1}$ & $1.1 \cdot 10^{16}$ & $2.5 \cdot 10^{14}$ & $2.6 \cdot 10^{14}$ \\
\hline Target material & $1 \mathrm{Hg}$ & $\mathrm{Hg}$ & $\mathrm{lHg}$ \\
\hline Collection system & Horn & Horn & Solenoid \\
\hline Energy range/MeV & $100-300$ & $100-180$ & \\
\hline Phase rotation & RF & - & Ind.linac. \\
\hline Cooling factor & & 1.0 & 3.4 \\
\hline Efficiency $\mu / \mathrm{pGeV}$ & 0.004 & 0.006 & 0.007 \\
\hline Muon energy/GeV & 50 & 20 & 20 \\
\hline Muon flux/detector/y ${ }^{-1}$ & $2.8 \cdot 10^{20}$ & $1.0 \cdot 10^{20}$ & $1.2 \cdot 10^{20}$ \\
\hline
\end{tabular}

Tab. 1 shows a comparison of the main parameters of three $\nu$-factory concepts that are being pursued in Europe [1], the US [2] and Japan [3]. It may be seen that there is quite a variety in the approaches. The choice of the proton source, its energy and repetition rate is determined mostly by what type of machine is considered to become available most easily in the region, by upgrading existing equipment. However, the proton beam powers are rather similar. The proton pulse length is of the order of $1 \mathrm{~ns}$. High $Z$ targets are favoured because they have a higher $\pi$ yield than low $Z$ material. The pions leave the target with a large spread in angle and longitudinal momentum. Collection systems using either magnetic horns or solenoids are being considered. Phase rotation uses the fact that muons with higher momentum $p$ arrive sooner at a distance $s$ from the target than muons with lower $p$, and decelerates the former and accelerates the latter by a time-varying accelerating field in an induction linac or RF cavities, such that the longitudinal momentum spread is reduced, and becomes suitable for subsequent acceleration. At the same time, the muon beam is bunched such that the bunches are short enough and separated in time by a multiple of the RF period in the acceleration system. The cooling factor is the ratio of the number of muons that arrive at the end of the cooling channel within the acceptance, with and without ionization cooling. In 
the Japanese neutrino factory, it is unity by definition, since there is no cooling. The efficiencies, i.e. the number of muons, arriving in the muon storage ring, per proton incident on the target and per $\mathrm{GeV}$ of proton energy, are rather similar in the three machines. The muon energies in the storage rings range form 20 to $50 \mathrm{GeV}$. The muon fluxes per distant detector in a year with $10^{7} \mathrm{~s}$ differ by less than a factor of three.

\section{FFAG - FIXED-FIELD-ALTERNATING-GRADIENT}

Acceleration of the muon beam in a cascade of fixed-field alternating-gradient synchrotrons is part of the Japanese $\nu$ factory [3]. The magnets in such a synchrotron are excited at constant fields. Bending and focusing are combined in the same magnets. Horizontally focusing and defocusing magnets have dipole fields in opposite directions. As a consequence, the horizontal dispersion is very small, and muons beams with momenta between $p_{i}$ at injection and $p_{e}$ at ejection, typically in the ratio 1:3, are within the same magnet aperture. The dipole field, averaged over the circumference of such a synchrotron, is much smaller than the dipole field in the magnets, the circumference is much larger than in a synchrotron with a FODO lattice, and the muon decay losses limit the number of turns. A few more tricks used in FFAG synchrotrons are beyond the scope of this summary.

\subsection{Motivation}

The proposal to use FFAG synchrotrons is motivated by the observation that the $\pi / \mu$ beam is produced in a 6D phase space of "large" volume. Hence, the $\pi / \mu$ emittances are much larger than the acceptance of typical strong focusing synchrotrons, operating at $\mathrm{RF}$ wavelengths $\lambda \approx 1 \mathrm{~m}$ with beam port radii, and physical apertures, that are only a fraction of $\lambda$. The authors of [3] claim that FFAG rings can be operated at $\lambda \geq 10 \mathrm{~m}$, and hence can have larger acceptances, and do not need phase rotation, bunching, ionization cooling, the expenditure as well as the muon decay losses associated with these processes. The authors of [1] and [2] feel that the RF cavities for acceleration at $\lambda \geq 10 \mathrm{~m}$ are bulky and expensive, and have adopted phase rotation, bunching, and ionization cooling.

\subsection{Injection and Ejection}

It is too early for a definitive answer whether such an FFAG scheme will work. We only have the ingredients of an answer. Yokoi and Shirakabe [4] considered ejection from the first FFAG synchrotron. Ejection is initiated by a full-aperture kicker magnet with a rise time close to a revolution period, if there is only one bunch in the synchrotron. The trajectory of the ejected beam is complicated by the compact lattice with short magnets and straight sections. The muon beam leaves the synchrotron aperture across the fringe field along outer edge of the magnets. The kick angle in the fast kicker magnet is proportional to $1 / p$. Hence, the spread in $\delta p / p$ causes a spread in the slopes 
of the trajectories that must be compensated downstream, if one does not want it to propagate through the remaining FFAG synchrotrons.

Extrapolating these results for ejection to injection into an FFAG synchrotron, I arrive at the following observations. The fast full-aperture kicker magnet is similar to the ejection kicker. It is advantageous that the kick angle is larger in the ratio $p_{e} / p_{i}$, making it easier to separate injected and circulating beam. It has the disadvantage that the injected beam size is larger in the ratio $\sqrt{p_{e} / p_{i}}$, neglecting variations of the focusing parameters of the FFAG lattice between injection and ejection energies, making it more difficult to separate injected and circulating beam. It also has the disadvantage that the spread in kick angles is larger due to the larger momentum spread $\delta p / p$ in the injected beam. By matching the injection beam line to the FFAG lattice with $D \neq 0$ and $D^{\prime} \neq 0$, one must ensure that muons with all momenta within the momentum spread $\delta p / p$ arrive on their proper orbit.

\subsection{Dynamic Aperture of FFAG Synchrotrons}

The horizontal dynamic aperture in a FFAG synchrotron was computed by Aiba and Yoshimoto [5], by tracking particles without synchrotron oscillations and vertical betatron. They found a value of about $30 \pi \mathrm{mm}$, only about 2 times larger than the design values of the European and US machines. The horizontal dynamic aperture will decrease when synchrotron and vertical betatron oscillations or $\delta p / p \neq 0$ are included in the simulation. For a definitive answer on the dynamic aperture of FFAG synchrotrons we should wait for tracking results of ensembles of $10^{4}$ to $10^{5}$ muons with realistic initial distributions in 6D, and realistic errors on alignment, excitation and field shape.

\subsection{Cooling in FFAG Synchrotrons}

Schönauer [6] considered mild cooling in the low-energy FFAG synchrotron. It would reduce the transverse emittances, and hence the vertical aperture, of the downstream FFAG synchrotrons and the muon storage ring. It has little effect on the horizontal aperture of the downstream FFAG synchrotrons, given by the momentum swing. It would slow down the acceleration, and enhance the muon decay losses. Schönauer concluded that such mild cooling is done better in a cooling insertion than all around the ring.

\subsection{Computational Techniques}

Talks about computational techniques were contributed by Johnstone [7], and Machida and Forest [8]. 


\section{TARGETTING AND COLLECTION}

The favoured target is a liquid $\mathrm{Hg}$ jet. In contrast to solid targets made of high- $Z$ material, it is believed to handle the proton beam parameters in Tab. 1. Radioactive isotopes from nuclear interactions either have a short lifetime or can be separated by distillation. Experiments with liquid $\mathrm{Hg}$ jets have started, as will be discussed in Chapter 5. The Neutrino Factory Study 2 at BNL [9] proposes an iron end plate and a nozzle at a high magnetic field inside the solenoid, thus avoiding possible magnetohydrodynamics problems arising from an unconstrained moving conductor entering a magnetic field.

\subsection{A Stationary Granular Target}

Sievers gives a schematic diagram of a stationary granular target in Fig. 1 of [10], and discusses its merits. He prefers a high proton energy, a high repetition rate, a large beam radius, and a long burst duration, just as every target designer. Tab. 2 shows a comparison of the proton beam parameters, and of the resulting target parameters for three neutrino factories.

\subsection{Neuffer's High-Frequency Adiabatic Buncher}

Tab. 1 already showed that the three neutrino factory proposals differ by the collection system, either a horn or a solenoid, and by the system for phase rotation, either a linear induction accelerator or RF cavities, operating at several harmonically related frequencies. An induction linac has the desirable feature that the pulse shape can be chosen as needed. It has the undesirable features that it is long, with the associated muon decay losses, expensive [11], and has not been used in a production facility, that should run reliably for at least $10^{7} \mathrm{~s}$ in a year. These features are a good reason for looking for alternatives. Neuffer [17] proposed one of them. He bunches the muon beam first with an adiabatic buncher whose frequency changes from $300 \mathrm{MHz}$ at entrance to $200 \mathrm{MHz}$ at exit, and then phase rotates with a $200 \mathrm{MHz}$ RF system.

\section{COOLING}

Muon cooling is a rapidly developing topic. Geer [12] reviewed muon cooling R\&D in a plenary talk. I shall not describe details that will change as result of NuFact'01. Hanke [13] presented the status of a cooling experiment at CERN. All cooling experiments will have to measure changes in the emittance of the muon beam to an accuracy better than a fraction of a percent. The choice is between two methods, one usually used in accelerators and storage rings, the other in particle detectors. The former method measures beam profiles with monitors, and uses the knowledge of the beam optics between the monitors to obtain the emittances. The latter method measures muon 
Table 2: Comparison of proton beam and target parameters for three neutrino factory proposals. The upper half of the table shows the absolute beam parameters. The lower part shows target parameters scaled to the parameters of the CERN neutrino factory. The average longitudinal power density, taken at the peak of the nuclear cascade, is a measure of how difficult is radial cooling. The instantaneous temperature rise in the target $\Delta T_{T}$ is taken at the peak of the nuclear cascade. $\Delta \sigma_{T}$ measures the shock in the target. It is roughly proportional to the ratio of $\Delta T_{T}$ and burst duration. $\Delta T_{W}$ measures the instantaneous temperature rise in the window and $\Delta \sigma_{W}$ the stress, directly proportional to it. This is a little subtle: The small spheres do not suffer much from shock, i.e. negligible or not severe, but still profit from longer pulse duration, while the wide window suffers from shock and does not profit much from longer pulse duration.

\begin{tabular}{lccc}
\hline & CERN & USA & Japan \\
\hline Beam power/MW & 4 & 4 & 1 \\
Beam energy/GeV & 2 & 24 & 30 \\
Rep. Rate/Hz & 50 & 50 & 0.5 \\
Beam size $\sigma / \mathrm{mm}$ & 10 & 5 & 5 \\
Burst duration/ $\mu \mathrm{s}$ & 3.3 & 0.003 & 10 \\
\hline$N_{p}^{+} / \mathrm{s}$ & 1 & 0.08 & 0.016 \\
$N_{p}^{+} /$Burst & 1 & 0.08 & 1.6 \\
Av. long. power density & 1 & 0.25 & No cooling problem \\
$\Delta T_{T}$ & 1 & 1.0 & 27 \\
$\Delta \sigma_{T}$ & 1 negligible & 10 not severe & $27 \cdot 3.3 \mu \mathrm{s} / 10 \mu \mathrm{s}=9$ \\
$\Delta T_{W} \approx \Delta \sigma_{W}$ & 1 & 0.33 & 6.4 \\
\hline \multicolumn{4}{c}{} \\
\hline
\end{tabular}

tracks, reconstructs the phase space coordinates, and obtains the emittances from large ensembles of muon tracks, using the knowledge of the electro-magnetic fields between the tracking devices. Chapter 5 discusses plans for a cooling experiment developed at NuFact'01.

\subsection{Moment Equations}

Penn [14] and Wang and Kim [15] set up and solve the equations of motion for the moments of the particle distribution functions in a muon cooling channel. They find reasonable agreement with multi-particle simulations. Since the number of moments is considerably smaller than the number of particles in a multi-particle simulation, parameter searches using the moment equations should be considerably faster than multi-particle simulations, even when the computer time per moment is larger than that per particle. I ask myself why no contribution to NuFact'01 reported parameter searches with moment equations.

Penn [14] and Wang and Kim [15] also include longitudinal muon cooling that is essential for $\mu^{+} \mu^{-}$colliders. The only known procedure for longitudinal cooling uses a wedge-shaped liquid hydrogen absorber, installed in a section where the dispersion 
is arranged such that muons with higher energy travel through the thicker side of the absorber.

\section{PROGRESS SINCE NUFACT'00}

Since NuFact'01, there has been progress on several fronts. The Neutrino Factory Study 2, completed at BNL [9], enhanced our understanding. We now believe that a $\nu$ factory is feasible, provided that the components behave as predicted in our simulations, and that the few things that we have neither simulated nor engineered give no surprises. Our US colleagues tell us Europeans and Japanese to carry out studies of similar scope and duration. Several workshops have taken place since NuFact'00. They are an excellent means of progressing on specific issues. An Emittance Exchange Workshop is scheduled at LBNL for first two weeks in October 2001, shortly after this summary will be submitted.

The particle and accelerator physics issues of $\mu^{+} \mu^{-}$colliders were presented by Hanson [16]. Fig. 9 in [16] shows Balbekov's ring cooler. Ring coolers hold the promise of achieving a given emittance reduction with fewer RF cavities and liquid hydrogen absorbers, because the muons make a few turns. Neuffer [17] proposed a scheme for injecting a muon beam with large longitudinal and transverse emittances into a ring cooler, and ejecting it.

Experiments and engineering are being done in international collaborations. We heard about several of them at NuFact'01, listed below in no particular order.

- The MUSCAT experiment [18] at TRIUMF measures multiple scattering of muons in various target materials. It hopes to be accurate enough to distinguish between theories for multiple scattering. MUSCAT had an engineering run with solid targets last year, and learned much about the experiment. MUSCAT will have a final run in 2001, also with liquid hydrogen.

- The HARP experiment [19] at CERN will measure particle production from protons between 2 and $15 \mathrm{GeV}$ in various targets. It was expected to start running within days of NuFact'01.

- The liquid Hg target experiment at BNL [20] has presented first results on the interaction between a liquid $\mathrm{Hg}$ jet and a short proton bunch. It will continue for a few years. The magneto-hydrodynamics of a liquid $\mathrm{Hg}$ jet entering a high solenoidal magnetic field might be studied at the National High Magnetic Field Laboratory in Tallahassee, USA and/or the High Magnetic Field Laboratory in Grenoble, France.

- Liquid hydrogen absorbers are being developed by a collaboration of laboratories in the US and Japan [21].

Ionization cooling is an important ingredient in the performance and cost of a neutrino factory. It has never been observed experimentally for minimum-ionizing muons. An earlier proposal for a muon cooling experiment [22] by the US muon collaboration 
has not been approved; a muon cooling experiment is a somewhat larger project than can be afforded by any one region. A step towards an International muon Cooling Experiment [23] was taken during NuFact'01. Its goal would be to design, engineer, and build a section of cooling channel that could become part of a high-performance neutrino factory design, to put it in a beam and to verify that it performs as expected. If it does not, understand why. It has been said that "The beam never lies". Such an experiment has also been called a string test, in analogy to the ongoing string tests of arc lattice cells for the LHC. The steering committee met at breakfast on 29 May 2001. It consists of A. Blondel (convener for 1 year), R. Edgecock, H. Haseroth from Europe, Y. Kuno, NN from Japan, and S. Geer, D. Kaplan, M. Zisman from the US. x The steering committee will constitute the technical teams [24]. It proposes the following agenda for the next few years:

2001 - Expose detectors to the abundant X rays, emitted by RF cavities, that could potentially preclude a single-particle experiment.

- Write first description of the experiment including options.

- US simulate CERN scheme

- Evaluate availability and/or cost of main cost drivers, i.e. RF cavities, power supplies, solenoids

- Evaluate beams and host laboratories

- First meeting on 25-27 October 2001 at CERN

- Choose technology and host laboratory, and write 15 pages Letter of Intent in December

2002 Submit technical proposal in Spring

2004 First Beam in Summer

\section{References}

[1] R. Garoby, European Neutrino Factory Studies, these proceedings.

[2] R. Palmer, US Neutrino Factory Studies, these proceedings.

[3] S. Machida, Japanese Neutrino Factory Studies, these proceedings.

[4] T. Yokoi and Y. Shirakabe, private communication (May 2001).

[5] M. Aiba and M. Yoshimoto, private communication (May 2001).

[6] H. Schönauer, Ionisation Cooling in FFAG Neutrino Factories, these proceedings.

[7] C. Johnstone, private communication (May 2001).

[8] S. Machida and E. Forest, private communication (May 2001).

[9] S. Ozaki, R. Palmer, M. Zisman and J. Gallardo (eds.), Feasibility StudyII of a Muon Based Neutrino Source, BNL-52623 (2001), also available at http://www.cap.bnl.gov/mumu/studyii/ 
[10] P. Sievers, A Stationary Target for the CERN Neutrino Factory, these proceedings.

[11] N. Holtkamp and D. Finley (eds.), A Feasibility Study of a Neutrino Source Based on a Muon Storage Ring, FERMILAB-PUB-00-108-E (2000).

[12] S. Geer, Muon Cooling $R \& D$, these proceedings.

[13] K. Hanke, Status of Studies for a Muon Cooling Experiment, these proceedings.

[14] G. Penn, private communication (May 2001).

[15] Chun-xi Wang and Kwang-Je Kim, Linear theory of transverse and longitudinal ionization cooling in a quadrupole channel, these proceedings.

[16] G. Hanson, Towards a Higgs Factory/Muon Collider, these proceedings.

[17] D. Neuffer, Injection and/or Extraction and a Ring Cooler, these proceedings.

[18] M. Ellis, Status of the MUSCAT Experiment, these proceedings.

[19] M.G. Catanesi et al., Proposal to study hadron production for the neutrino factory and for the atmospheric neutrino flux, CERN-SPSC/99-35 (1999).

[20] K. McDonald, Target $R \& D$, these proceedings.

[21] D. Kaplan, Liquid-Hydrogen Absorber $R \& D$, these proceedings.

[22] C.M. Ankenbrandt et al., Ionization Cooling Research and Development for a High Luminosity Muon Collider, Proposal P904, Fermilab (1998).

[23] http://hep04.phys.iit.edu/cooldemo/

[24] A. Blondel et al. An International Cooling Experiment: Goals and Preliminary Design, http://hep04.phys.iit.edu/cooldemo/intcoolexp.pdf 